\title{
Generalized discrete operators
}

\author{
RUI A. C. FERREIRA ${ }^{a}$ (i) \\ a Grupo Física-Matemática, Faculdade de Ciências, Universidade de Lisboa, Av. Prof. \\ Gama Pinto, 2, 1649-003, Lisboa, Portugal.
}

• Received: 07.06.2021 • Accepted: 18.06.2021 • Published Online: 29.06.2021

\begin{abstract}
We define a class of discrete operators that, in particular, include the delta and nabla fractional operators. Moreover, we prove the fundamental theorem of calculus for these operators.
\end{abstract}

Keywords: Discrete calculus, fractional operators.

2010 MSC: 39A12, 26A33.

\section{Preamble}

The theory of discrete fractional calculus is currently an area of mathematics of intensive research, having appeared in the literature many articles on the subject in the past decade (see $[1,2,3,4,5,6,7,8]$ and the references therein). Two parallel concepts were introduced, namely, the delta (or forward) operators and the nabla (or backward) operators (see [9, Sections 2 and 3]).

Consider the falling function defined, for $x, y \in A \subset \mathbb{R}$, by

$$
\chi \underline{y}=\left\{\begin{array}{l}
x(x-1) \ldots(x-y+1) \text { for } y \in \mathbb{N}_{1}, \\
1 \text { for } y=0 \\
\frac{\Gamma(x+1)}{\Gamma(x+1-y)} \text { for } x, x-y \notin \mathbb{N}^{-1} \\
0 \text { for } x \notin \mathbb{N}^{-1} \text { and } x-y \in \mathbb{N}^{-1}
\end{array}\right.
$$

and the rising function defined by

$$
x^{\bar{y}}=(x+y-1) \underline{y} .
$$

Then, letting $\mathbb{N}_{a}=\{a, a+1, \ldots\}$ with $a \in \mathbb{R}$ and $f: \mathbb{N}_{a} \rightarrow \mathbb{R}$ being a function, the delta Riemann-Liouville fractional sum of $f$ of order $v>0$ is defined by

$$
\Delta_{a}^{-v} f(t)=\sum_{s=a}^{t-v} \frac{(t-(s+1)) \underline{v-1}}{\Gamma(v)} f(s) \text { for } t \in \mathbb{N}_{a+v-1},
$$

*Corresponding author: raferreira@fc.ul.pt

(C) 2020 SABA. All Rights Reserved. 
while the nabla Riemann-Liouville fractional sum of $f$ of order $v>0$ is defined by

$$
\nabla_{a}^{-v} f(t)=\sum_{s=a+1}^{t} \frac{(t-(s-1))^{\overline{v-1}}}{\Gamma(v)} f(s) \text { for } t \in \mathbb{N}_{a} .
$$

One can observe that the above sums are of the type

$$
\sum k(t-s \pm 1) f(s),
$$

for a certain kernel function $k$. Now, if we consider the delta difference operator $\Delta f(t)=$ $f(t+1)-f(t)$ and the nabla difference operator $\nabla f(t)=f(t)-f(t-1)$, then the delta and nabla Riemann-Liouville fractional differences of $f$ of order $0<\alpha \leqslant 1$ are defined by,

$$
\Delta_{\mathrm{a}}^{\alpha} \mathrm{f}(\mathrm{t})=\Delta\left[\Delta_{\mathrm{a}}^{-(1-\alpha)} \mathrm{f}\right](\mathrm{t}), \mathrm{t} \in \mathbb{N}_{\mathrm{a}+1-\alpha,}
$$

and

$$
\nabla_{\mathrm{a}}^{\alpha} \mathrm{f}(\mathrm{t})=\nabla\left[\nabla_{\mathrm{a}}^{-(1-\alpha)} \mathrm{f}\right](\mathrm{t}), \mathrm{t} \in \mathbb{N}_{\mathrm{a}+1},
$$

respectively.

In this work we aim to construct a summation and a difference operator generalizing the above ones and satisfying the fundamental theorem of calculus (we are particularly inspired by the work of Kochubei [10] in which such kind of operators were defined for (continuous) integrals and derivatives). Hopefully, these very general operators will be useful for researchers acting within the discrete calculus theory.

\section{Main results}

Let us start by recalling the discrete convolution of two functions $f, g: \mathbb{N}_{a} \rightarrow \mathbb{R}$, with $a \in \mathbb{R}$ : it is denoted by $(\mathrm{f} * \mathrm{~g})_{a}$ and defined by

$$
(f * g)_{a}(t)=\sum_{\tau=a}^{t-1} f(t-\tau-1+a) g(\tau), \quad t \in \mathbb{N}_{a} .
$$

Here and throughout this text we assume that empty sums are equal to zero. Therefore, $(f * g)_{a}(a)=0$ for all functions $f$ and $g$. It is known that the convolution is commutative and associative (cf. [11, Theorem 5.4]).

Let us introduce the following set of pair-of-functions: For $a \in \mathbb{R}$, put

$$
\mathcal{C}_{\mathrm{a}}=\left\{\mathrm{p}, \mathrm{q}: \mathbb{N}_{\mathrm{a}} \rightarrow \mathbb{R}:(\mathrm{p} * \mathrm{q})_{\mathrm{a}}(\mathrm{t})=1 \text { for all } \mathrm{t} \in \mathbb{N}_{\mathrm{a}+1}\right\}
$$

Before we proceed, we state here the fractional power rule, whose proof may be found in [6].

Lemma 2.1. Let $a \in \mathbb{R}$. Assume $\mu \notin \mathbb{N}^{-1}$ and $v>0$. If $\mu+v \notin \mathbb{N}^{-1}$, then

$$
\Delta_{a+\mu}^{-v}[(s-a) \underline{\mu}](t)=\frac{\Gamma(\mu+1)}{\Gamma(\mu+v+1)}(t-a) \stackrel{\mu+v}{v}, \text { for } t \in \mathbb{N}_{a+\mu+v} .
$$


Example 2.2. Let $a \in \mathbb{R}$ and $\alpha \in \mathbb{R}^{+} \backslash \mathbb{N}_{1}$. Define the functions

$$
p(t)=\frac{(t-a) \underline{\alpha-1}}{\Gamma(\alpha)} \text { and } q(t)=\frac{(t-a+1-2 \alpha) \frac{-\alpha}{-}}{\Gamma(1-\alpha)}, \quad t \in \mathbb{N}_{a+\alpha-1} .
$$

Then, for all $t \in \mathbb{N}_{a+\alpha}$, we have

$$
\begin{aligned}
& (p * q)_{a+\alpha-1}(t)=\sum_{\tau=a+\alpha-1}^{t-1} \frac{(t-\tau-1+\alpha-1) \frac{\alpha-1}{(\tau-a+1-2 \alpha) \frac{-\alpha}{-}}}{\Gamma(\alpha)} \frac{(1-\alpha)}{\Gamma(1-\alpha)} \\
& =\sum_{\tau=\mathrm{a}}^{\mathrm{t}-\alpha} \frac{(\mathrm{t}-(\tau+1)) \frac{\alpha-1}{\Gamma(\alpha)}}{\Gamma(\tau-(\mathrm{a}+\alpha)) \frac{-\alpha}{\Gamma(1-\alpha)}} \\
& =\frac{\Delta_{\mathrm{a}}^{-\alpha}[(\tau-(\mathrm{a}+\alpha))-\alpha](\mathrm{t})}{\Gamma(1-\alpha)} \\
& =1 \text {, }
\end{aligned}
$$

where we used (2.1) to obtain the last equality. Hence, the pair $(p, q) \in \mathcal{C}_{a+\alpha-1}$.

Analogously, if we define the functions

$$
\hat{p}(t)=\frac{(t-a+1)^{\overline{\alpha-1}}}{\Gamma(\alpha)} \text { and } \hat{q}(t)=\frac{(t-a+1)^{\overline{-\alpha}}}{\Gamma(1-\alpha)}
$$

then we may show, upon using (1.1) and Lemma 2.1 , that $(\hat{p}, \hat{q}) \in \mathcal{C}_{a}$. Indeed,

$$
\begin{aligned}
& (\hat{p} * \hat{q})_{a}(t)=\sum_{\tau=a}^{t-1} \frac{(t-\tau)^{\overline{\alpha-1}}}{\Gamma(\alpha)} \frac{(\tau-a+1)^{\overline{-\alpha}}}{\Gamma(1-\alpha)} \\
& =\sum_{\tau=\mathrm{a}}^{\mathrm{t}-1} \frac{(\mathrm{t}+\alpha-1-(\tau+1)) \frac{\alpha-1}{\Gamma(\alpha)}}{\Gamma(\tau-(\mathrm{a}+\alpha)) \underline{-\alpha}} \\
& =\frac{\Delta_{\mathrm{a}}^{-\alpha}\left[(\tau-(\mathrm{a}+\alpha)) \frac{-\alpha}{\alpha}\right](\mathrm{t}+\alpha-1)}{\Gamma(1-\alpha)} \\
& =1 \text {, }
\end{aligned}
$$

Remark 2.3. A somewhat more elaborate example of a pair of functions belonging to $\mathfrak{C}_{0}$ may be found in [12, Example 9].

Definition 2.4. Let $p, q: D \subset \mathbb{R} \rightarrow \mathbb{R}$ be two functions. We define the generalized fractional sum (GFS) of $f: \mathbb{N}_{a} \rightarrow \mathbb{R}$ by

$$
\mathcal{S}_{\{(p) ; a\}} f(t)=\sum_{\tau=a}^{t-1} p(t-\tau-1+a) f(\tau), \quad t \in \mathbb{N}_{a} .
$$

Moreover, the generalized fractional difference of Riemann-Liouville type is defined by

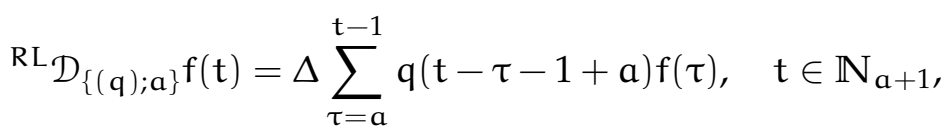


while the generalized fractional difference of Caputo type is defined by

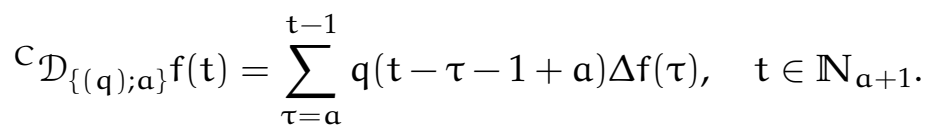

The previous definition includes the delta and nabla operators mentioned in Section 1. Indeed, first consider a function $f: \mathbb{N}_{a} \rightarrow \mathbb{R}$. Then,

- Consider $p(t)=\frac{(t-a) \underline{\alpha-1}}{\Gamma(\alpha)}$, for $t \in \mathbb{N}_{a+\alpha-1}$. Then, for $t \in \mathbb{N}_{a+\alpha-1}$, we have

$$
\begin{aligned}
\mathcal{S}_{\{(p) ; a+\alpha-1\}}[f(\tau+1-\alpha)](t) & =\sum_{\tau=a+\alpha-1}^{t-1} \frac{(t-(\tau+1)+\alpha-1) \frac{\alpha-1}{\Gamma(\alpha)}}{\Gamma(\tau+1-\alpha)} \\
& =\sum_{\tau=a}^{t-\alpha} \frac{(t-(\tau+1)) \frac{\alpha-1}{\Gamma(\alpha)}}{\tau} f(\tau) \\
& =\Delta_{a}^{-\alpha} f(t) .
\end{aligned}
$$

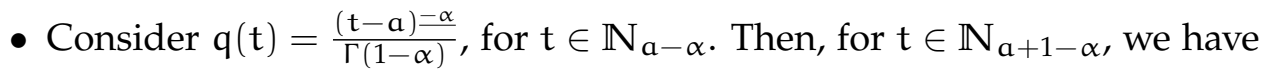

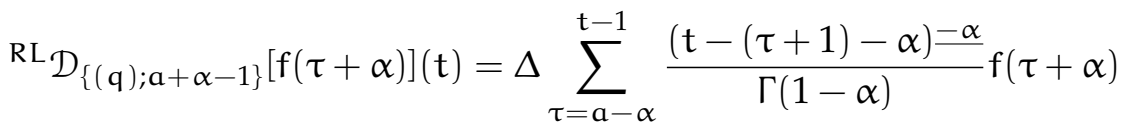

$$
\begin{aligned}
& =\Delta \sum_{\tau=a}^{t+\alpha-1} \frac{(t-(\tau+1)) \underline{-\alpha}}{\Gamma(1-\alpha)} f(\tau) \\
& =\Delta_{\mathrm{a}}^{\alpha} \mathrm{f}(\mathrm{t}) \text {. }
\end{aligned}
$$

Now, consider a function $f: \mathbb{N}_{\mathrm{a}+1} \rightarrow \mathbb{R}$. Then,

- Consider $\hat{p}(t)=\frac{(t-a+1)^{\overline{\alpha-1}}}{\Gamma(\alpha)}$, for $t \in \mathbb{N}_{a}$. Then, for $t \in \mathbb{N}_{a}$, we have

$$
\begin{aligned}
\mathcal{S}_{\{(\hat{p}) ; a\}}[f(\tau+1)](t) & =\sum_{\tau=a}^{t-1} \frac{(t-\tau)^{\overline{\alpha-1}}}{\Gamma(\alpha)} f(\tau+1) \\
& =\sum_{\tau=a+1}^{t} \frac{(t-(\tau-1))^{\overline{\alpha-1}}}{\Gamma(\alpha)} f(\tau) \\
& =\nabla_{a}^{-\alpha} f(t) .
\end{aligned}
$$

- Consider $\hat{\mathrm{q}}(\mathrm{t})=\frac{(\mathrm{t}-\mathrm{a}+1)^{-\alpha}}{\Gamma(1-\alpha)}$, for $\mathrm{t} \in \mathbb{N}_{\mathrm{a}}$. Then, for $\mathrm{t} \in \mathbb{N}_{\mathrm{a}+1}$, we have

$$
\begin{aligned}
\mathrm{RL}_{\mathcal{D}_{\{(\hat{\mathrm{q}}) ; \mathrm{a}+1\}}[\mathrm{f}](\mathrm{t})} & =\Delta \sum_{\tau=\mathrm{a}+1}^{\mathrm{t}-1} \frac{(\mathrm{t}-\tau)^{-\alpha}}{\Gamma(1-\alpha)} \mathrm{f}(\tau) \\
& =\sum_{\tau=\mathrm{a}+1}^{\mathrm{t}} \frac{(\mathrm{t}+1-\tau)^{-\alpha}}{\Gamma(1-\alpha)} \mathrm{f}(\tau)-\sum_{\tau=\mathrm{a}+1}^{\mathrm{t}-1} \frac{(\mathrm{t}-\tau)^{-\alpha}}{\Gamma(1-\alpha)} f(\tau) \\
& =\nabla_{\mathrm{a}}^{\alpha} \mathrm{f}(\mathrm{t}) .
\end{aligned}
$$


To prove our main result we need the following (cf. [9, Theorem 1.67]):

Proposition 2.5 (Leibniz rule). Assume $\mathrm{f}: \mathbb{N}_{\mathrm{a}+1} \times \mathbb{N}_{\mathrm{a}} \rightarrow \mathbb{R}$. Then,

$$
\Delta_{t} \sum_{s=a}^{t-1} f(t, s)=\sum_{s=a}^{t-1} \Delta_{t} f(t, s)+f(t+1, t), \quad t \in \mathbb{N}_{a} .
$$

It follows the fundamental theorem of calculus for these generalized discrete operators.

Theorem 2.6. Let $\mathrm{a} \in \mathbb{R}$ and suppose that $(\mathrm{p}, \mathrm{q}) \in \mathcal{C}_{\mathrm{a}}$. Then,

$$
{ }^{R L} \mathcal{D}_{\{(q) ; a\}} \mathcal{S}_{\{(p) ; a\}} f(t)={ }^{C} \mathcal{D}_{\{(q) ; a\}} \mathcal{S}_{\{(p) ; a\}} f(t)=f(t), \quad t \in \mathbb{N}_{a+1} .
$$

Moreover,

$$
\mathcal{S}_{\{(p) ; a\}}{ }^{R L} \mathcal{D}_{\{(q) ; a\}} f(t)=f(t), \quad t \in \mathbb{N}_{a}
$$

and

$$
\mathcal{S}_{\{(p) ; a\}} C_{\mathcal{D}_{\{(q) ; a\}} f(t)=f(t)-f(a), \quad t \in \mathbb{N}_{a} .}
$$

Proof. Since $(p, q) \in \mathcal{C}_{a}$ we know that

$$
(p * q)_{a}(t)=1 \text { for all } t \in \mathbb{N}_{a+1} .
$$

It follows that,

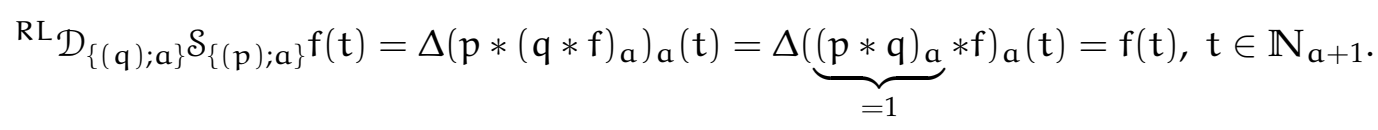

Now, by the Leibniz rule, we have that

$$
{ }^{R L} \mathcal{D}_{\{(q) ; a\}} f(t)=(q * \Delta f)_{a}(t)+f(a) q(t)={ }^{C} \mathcal{D}_{\{(q) ; a\}} f(t)+f(a) q(t) .
$$

Since $\mathcal{S}_{\{(p) ; a\}} f(a)=0$, we immediately conclude that ${ }^{C} \mathcal{D}_{\{(q) ; a\}} \mathcal{S}_{\{(p) ; a\}} f(t)=f(t)$ and, consequently, (2.2) is proved.

To prove (2.3), we observe that

$$
\begin{aligned}
\mathcal{S}_{\{(p) ; a\}}{ }^{R L} \mathcal{D}_{\{(q) ; a\}} f(t) & =\mathcal{S}_{\{(p) ; a\}}\left[(q * \Delta f)_{a}(t)+f(a) q(t)\right] \\
& =(1 * \Delta f)_{a}+f(a)(p * q)_{a}=f(t) .
\end{aligned}
$$

Finally, (2.4) follows from

$$
\begin{aligned}
\mathcal{S}_{\{(p) ; a\}}{ }^{C} \mathcal{D}_{\{(q) ; a\}} f(t) & \left.=\mathcal{S}_{\{(p) ; a\}}{ }^{[R L} \mathcal{D}_{\{(q) ; a\}} f-f(a) q\right] \\
& =f(t)-f(a)(p * q) a \\
& f(t)-f(a) .
\end{aligned}
$$

The proof is done.

\section{Acknowledgement}

Rui A. C. Ferreira was supported by the "Fundação para a Ciência e a Tecnologia $(\mathrm{FCT})$ " through the program "Stimulus of Scientific Employment, Individual Support2017 Call" with reference CEECIND/00640/2017. 


\section{References}

[1] Abdeljawad T (2013). On delta and nabla Caputo fractional differences and dual identities. Discrete Dyn. Nat. Soc. 2013, Art. ID 406910, 12 pp. https:/ / doi.org/10.1155/2013/406910

[2] Atici FM, Eloe PW (2007). A transform method in discrete fractional calculus. Int. J. Difference Equ. 2(2): 165-176. https: / /ecommons.udayton.edu/mth_fac_pub/110

[3] Čermák J, Győri I, Nechvátal L (2015). On explicit stability conditions for a linear fractional difference system. Fract. Calc. Appl. Anal. 18(3): 651-672. https://doi.org/10.1515/fca-2015-0040

[4] Ferreira RAC (2012). A discrete fractional Gronwall inequality. Proc. Amer. Math. Soc. 140(5): 1605-1612. https:/ / www.jstor.org/stable/41505615

[5] Ferreira RAC (2021). Discrete weighted fractional calculus and applications. Nonlinear Dyn. 104: 2531-2536. https:/ / doi.org/10.1007/s11071-021-06410-6

[6] Ferreira RAC (2020). Discrete fractional calculus and the Saalschutz theorem. arXiv preprint, arXiv:2008.11605v1.

[7] Gray HL, Zhang NF (1988). On a new definition of the fractional difference. Math. Comp. 50 (182): 513-529. https: / doi.org/10.1090/S0025-5718-1988-0929549-2

[8] Miller KS, Ross B (1988). Fractional difference calculus, in Univalent functions, fractional calculus, and their applications. Koriyama, 139-152, Ellis Horwood Ser. Math. Appl, Horwood, Chichester.

[9] Goodrich C, Peterson AC (2015). Discrete fractional calculus. Springer, Cham.

[10] Kochubei AN, (2011). General fractional calculus, evolution equations, and renewal processes. Integr. Equ. Oper. Theory 71 (4): 583-600.https:/ / doi.org/10.1007/s00020-011-1918-8

[11] Bohner M, Guseinov GS (2007). The convolution on time scales. Abstr. Appl. Anal. 2007 Art. ID 58373: 24 pp. https://doi.org/10.1155/2007/58373

[12] C. Goodrich C, Lizama C (2020). Positivity, monotonicity, and convexity for convolution operators. Discrete Contin. Dyn. Syst. 40(8): 4961-4983. https://doi: 10.3934/dcds.2020207 